\title{
Gait and Dynamic Balance Sensing Using Wearable Foot Sensors
}

\author{
Mohamed Irfan Mohamed Refai, Bert-Jan F. van Beijnum, Member, IEEE, Jaap H. Buurke, \\ and Peter H. Veltink, Senior Member, IEEE
}

\begin{abstract}
Remote monitoring of gait performance offers possibilities for objective evaluation, and tackling impairment in motor ability, gait, and balance in populations such as elderly, stroke, multiple sclerosis, Parkinson's, etc. This requires a wearable and unobtrusive system capable of estimating ambulatory gait and balance measures, such as Extrapolated Centre of Mass (XCoM) and dynamic Margin of Stability (MoS). These estimations require knowledge of 3D forces and moments (F\&M), and accurate foot positions. Though an existing Ambulatory Gait and Balance System (AGBS) consisting of 3D F\&M sensors, and inertial measurement units (IMUs) can be used for the purpose, it is bulky and conspicuous. Resistive pressure sensors were investigated as an alternative to the onboard 3D F\&M sensors. Subject specific regression models were built to estimate 3D F\&M from 1D plantar pressures. The model was applicable for different walking speeds. Different pressure sensor configurations were studied to optimise system complexity and accuracy. Using resistive sensors only under the toe and heel, we were able to estimate the XCoM with a mean absolute RMS error of $2.2 \pm 0.3 \mathrm{~cm}$ in the walking direction while walking at a preferred speed, when compared to the AGBS. For the same case, the XCoM was classified as ahead or behind the Base of Support correctly at $97.7 \pm 1.7 \%$. In conclusion, the study shows that pressure sensors, minimally under the heel and toe, offer a lightweight and inconspicuous alternative for $\mathrm{F} \& M$ sensing, towards estimating ambulatory gait and dynamic balance.
\end{abstract}

Index Terms-Ambulatory sensing, Extrapolated Center of Mass, Dynamic Balance, Margin of Stability, Gait, Wearable Sensors

\section{INTRODUCTION}

A SSESSMENT of motor impairment periodically during rehabilitation is crucial in understanding recovery. This is feasible via clinical outcome measures, and instrumented laboratory facilities, particularly in subjects with motor impairment, such as stroke, Parkinson's, multiple sclerosis, etc., and also others prone to instability such as frail elderly. Clinical outcomes indicate a subjective change in capacity or function of given tasks whereas instrumented systems offer objective quantification of kinematic and kinetic changes on impairment level of said tasks. Once the patient is discharged from the

Manuscript received MONTH DATE, YEAR; revised MONTH DATE, YEAR.

This work is part of the Perspectief programme NeuroCIMT with project number 14905 which is (partly) financed by the Netherlands Organisation for Scientific Research (NWO).

M. I. Mohamed Refai is with Biomedical Signals and Systems, Universiteit Twente, Enschede, 7522NB, The Netherlands e-mail: m.i.mohamedrefai@utwente.nl

J. H. Buurke is with Roessingh Research and Development and Biomedical Signals and Systems, Universiteit Twente, Enschede, The Netherlands

B. F. van Beijnum and P. H. Veltink are with Biomedical Signals and Systems, Universiteit Twente. clinic, she/he is expected to continue functional training to maintain recovery and increase independence in activities of daily life (ADL) [1]. Continuing objective monitoring after discharge helps quantify recovery, and identify compensatory patterns if present [2], [3]. As instrumented laboratory facilities are expensive and restrictive in measurement space, wearable systems can be explored as alternatives.

ForceShoe $^{\mathrm{TM}}$ (Xsens Technologies B.V., Enschede, The Netherlands) is a shoe with onboard sensors and was developed as an ambulatory gait lab [4], [5]. It consists of two Inertial Measurement Units (IMUs) and two 3D force and moment $(\mathrm{F} \& \mathrm{M})$ sensors on each foot. It has been validated against commonly used systems, such as force plates (AMTI ${ }^{\circledR}$ ) and motion capture $\left(\mathrm{VICON}^{\circledR}\right)$, for measurement of contact forces and foot position estimations respectively [5]-[7]. Unlike these systems, ForceShoe ${ }^{\mathrm{TM}}$ has the advantage of being portable, and not restricted by area of measurement setup or marker placement. It is used to reconstruct the kinematics and kinetics of the feet during walking [8]-[10].

Gait measures such as step length and width, and foot positions can be estimated using IMUs on the ForceShoe ${ }^{\mathrm{TM}}$. Weenk et al. [5], reduced the drift in position estimation using an Extended Kalman Filter (EKF), and validated it with motion capture. The EKF fused foot positions from the IMUs, and relative foot distance from an ultrasound sensor. The ForceShoe ${ }^{\mathrm{TM}}$, along with ultrasound sensor, EKF, and additional processing will be referred to as Ambulatory Gait and Balance System (AGBS).

Using the AGBS, Schepers et al. [6] derived Centre of Mass $(\mathrm{CoM})$ of the body during gait. Subsequently, van Meulen et al. [9] estimated the Extrapolated Centre of Mass (XCoM), which is the CoM extrapolated in the direction of the walking velocity. The trajectory of the XCoM projected on the ground with respect to the Base of Support (BoS), which is the region between two feet when they are in contact with the ground, is an indication of dynamic stability [11]. van Meulen et al. [9] used the shortest distance from the XCoM and the frontline of the BoS as a condition of stability during continuous gait, called as the dynamic Margin of Stability (MoS) [11]. Bruijn et al. [12], suggested that XCoM (and eventually MoS) can objectively indicate balance quality, and van Meulen et al. [9] showed that MoS varies with differences in subject's balance impairments. Balance quality is otherwise estimated using clinical outcomes such as Berg Balance Scale, Dynamic Gait Index, Tinetti Falls Scale, etc. However, the construct of these outcomes show that they are subjective to the assessor. Using a system like AGBS, one can objectively 
evaluate gait and dynamic balance in an ambulatory or home setting for different populations including stroke, multiple sclerosis, Becker's Muscle Dystrophy, Parkinson's, elderly, etc.

However, the system has its limitations. Liedtke et al. [7], showed that gait pattern is slightly modified when wearing the AGBS. Moreover, it is not practical to use them every day due to the bulky 3D F\&M sensors. These make each shoe weigh close to $1 \mathrm{~kg}$ and increases the sole height by $2.5 \mathrm{~cm}$ [9], increasing discomfort and chances of tripping, especially for people with gait impairments. Alternative systems for ambulatory estimation of 3D F\&M can be achieved by using an IMU-only setup. Karatsidis et al. solved this using 17 IMUs placed in a full body suit [13], [14]. However, the system requires a full body setup and the effect of a reduced IMU setup for this purpose has not yet been studied.

Insoles with pressure sensors can be used as an alternative to the bulky 3D F\&M sensors. They can be slipped into everyday use shoes, are lightweight, and inconspicuous. They provide 1D plantar pressures under the feet during walking and can be used for estimating a range of gait parameters [15]-[17].

Pressure insoles can only provide the vertical plantar pressure. However, 3D F\&M of the feet (along with its positions) are required to estimate the CoM, XCoM and MoS [6]. Studies have shown estimations of 3D F\&M from 1D plantar pressures by using analytic and machine learning methods. Forner-Cardeno et al. [18] showed analytic derivation of 3D forces from 1D plantar pressure data, but his method relies on force plate data. Sim et al. [19], showed the use of a wavelet neural network to estimate time-normalised 3D F\&M from 1D plantar pressure. Other studies use other machine learning methods to achieve the same [20]-[23]. In spite of a few disadvantages, such as the need for training data, or possible failure in untrained scenarios, machine learning methods have some potential use in this study.

The goal of this study is to evaluate 1D plantar pressure sensing as a lightweight alternative to the 3D F\&M sensors in AGBS for estimating dynamic balance measures. To do so, linear regression models were built to predict 3D F\&M from 1D plantar pressures. The models are subject specific and independent of walking speeds. The predicted 3D F\&M along with foot positions are used to estimate CoM, XCoM, and MoS. The estimations are compared with those of the AGBS for different pressure sensor configurations. The study throws light on pressure sensors as a replacement to the 3D F\&M sensors, the influence of the number of sensors on the estimation of $\mathrm{XCoM}$ and $\mathrm{MoS}$, and insights on the algorithms used during the process.

\section{Methods}

\section{A. Measurement System}

Fig. 1(a) shows the ForceShoe ${ }^{\mathrm{TM}}$ containing two 3D F\&M sensors, two IMUs, and an ultrasound. Only the IMU located at the forefoot was used for analysis. The data from the 3D F\&M sensors and IMUs were sent to an Xbus that transmits data wirelessly to a PC. The transmitter and receiver of the ultrasound system was placed on the right and left foot respectively. They were synchronized and the data were transmitted via Bluetooth to a PC. Fig. 1(b) shows the pressure insole system (medilogic ${ }^{\circledR}$ insoles, T\&T medilogic Medizintechnik $\mathrm{GmbH}$, Germany) placed in the ForceShoe ${ }^{\mathrm{TM}}$. It has 151 resistive pressure sensors and was held in place using tape to eliminate slippage. The wireless transmitters of the pressure insoles and ForceShoe ${ }^{\mathrm{TM}}$ were worn as a belt around the waist. The 3D F\&M sensors, IMUs, and the pressure insoles were sampled at $50 \mathrm{~Hz}$. The data was then low pass filtered twice at $10 \mathrm{~Hz}$ using second order butterworth filter to ensure zero-phase lag. The data was then transformed to the global coordinate frame, where $\mathrm{X}$ axis is along the walking direction and $\mathrm{Z}$ axis is the vertical axis pointing upwards.

\section{B. Participants}

Six healthy subjects were recruited for the study. All participants signed an informed consent before the experiment. The study was conducted in accordance with the Declaration of Helsinki, and the protocol was approved by the Ethical Committee of the faculty. The inclusion criteria included subjects with no history of impaired gait or leg injury. Five subjects were males and the size of the shoe used was 44 (European Size Chart). The average and standard deviation of the height, weight, and age was $1.81 \pm 0.06 \mathrm{~m}, 81 \pm 9 \mathrm{~kg}$, and $25 \pm 1$ years respectively. Leg length was measured from the greater trochanter to the ground [24] and was $1.04 \pm 0.05 \mathrm{~m}$.

\section{Experimental Protocol}

The ultrasound system and 3D F\&M sensors were calibrated before each measurement. The subjects were then asked to perform different walking tasks. In each of the walking tasks, the subject was asked to walk for 10 metres along a straight unobstructed path. The subject was instructed to begin with their feet placed parallel. Once the researcher gave the start sign, the subject walked along a straight line. The time taken between start and stop of the walking was measured using a stopwatch. This activity was repeated six times. The walking tasks were performed in four different scenarios which are as follows:

1) Normal walking: During this task, the subject was asked to walk at his preferred walking speed.

2) Slow walking: During this task, the subject was asked to walk at a slower pace. The speed was guided by the use of a metronome that beat at a frequency of 37 beats per minute. Each beat corresponds to heel strike of the same foot on the ground. The frequency was found using trial and error such that the subjects reduced their walking speed to $0.8 \mathrm{~m} / \mathrm{s}$.

3) Very Slow walking: During this task, the subject was asked to walk at a much slower pace. The frequency of the metronome was set at 27 beats per minute. This frequency was found so as to reduce the walking speed to $0.4 \mathrm{~m} / \mathrm{s}$.

4) Bag walking: During this task, the subject was asked to walk at his preferred walking speed while wearing a backpack weighing $5 \mathrm{~kg}$. This is to represent an extra, yet minimal load people may carry during daily life tasks, such as a shopping bag. 
a)

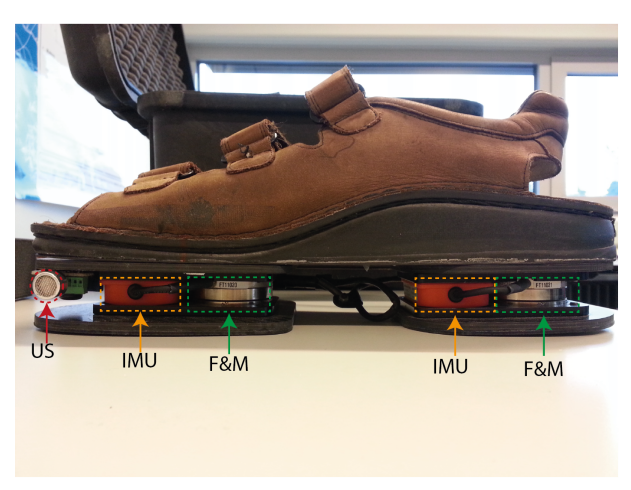

b)

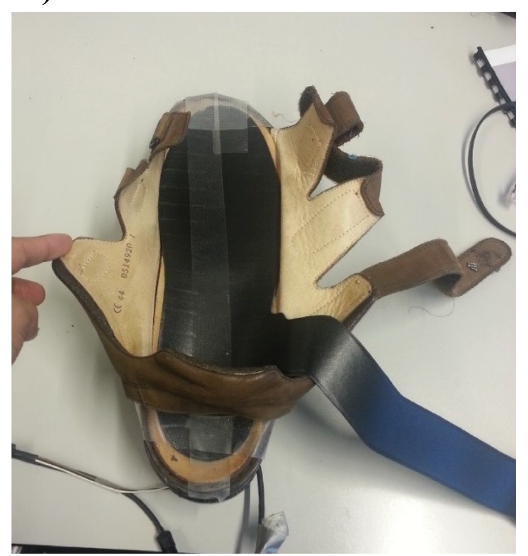

Fig. 1. (a) Side view of right ForceShoe. The ultrasound transmitter (US), the 3D F\&M sensors (F\&M), and inertial measuring units (IMU) are seen on the side. (b) Top down view of the ForceShoe fitted with pressure insole.

\section{Objective Evaluation of Gait and Dynamic Balance}

1) Gait: Ultrasound and IMUs were used for estimating foot positions, from which gait parameters such as step length, and step width were obtained. The EKF predicted states of position, instantaneous velocity, orientation error and gyroscope bias error [5, Fig. 3]. Error between predicted and measured data was used to correct the states for every measurement sample. Measurement updates included foot position and instantaneous velocity measured from the IMU, zero velocity instances, height of IMU during zero velocity and relative feet distance from the ultrasound system [5]. The ultrasound updates were used in the EKF at an update frequency of $13 \mathrm{~Hz}$ [9, Fig. 2].

2) Dynamic Balance: Estimation of CoM is the first step towards evaluating dynamic balance. Low and high frequency components of CoM were estimated using two separate algorithms and fused using a complementary filter, to improve estimation accuracy [6]. The first stage estimates CoM from both foot kinetic and kinematic information by low pass filtering the Centre of Pressure $(\mathrm{CoP})$ to estimate the position of CoM, referred to as Stage Low Frequency (Stage LF). Here, the $\mathrm{CoP}$ for each foot is estimated as follows.

$$
\mathbf{x}_{C o P, f o o t}=\left(\frac{-M_{Y}}{F_{Z}}, \frac{M_{X}}{F_{Z}}, 0\right)
$$

In Equation $1, F_{Z}$ is the vertical ground reaction force (GRF), and $M_{Y}$ and $M_{X}$ denote the moments in the respective axes. The CoP trajectory over the walking trial was weighted with the relative magnitude of the vertical GRF under each foot and is given as follows.

$$
\mathbf{x}_{C o P}=\frac{F_{l}}{F_{l}+F_{r}} \mathbf{x}_{C o P, l}+\frac{F_{r}}{F_{l}+F_{r}} \mathbf{x}_{C o P, r}
$$

Here, the $F_{l}$ and $F_{r}$ represent the vertical GRF in the left and right foot respectively. The $\mathbf{x}_{C o P}$ was then low pass filtered at $0.4 \mathrm{~Hz}$ to obtain the $\mathbf{x}_{C o M, L F}$. The cut off was found to be optimal for continuous walking [6]. The Stage LF CoM is estimated from both foot kinetic and kinematic information.
The second algorithm estimates $\mathrm{CoM}$ from kinetic information alone by double integration of the net forces based on Newton's second law, referred hereafter as Stage High Frequency (Stage HF). The body mass $m_{\text {body }}$ can be embodied at the CoM and it's acceleration is given as follows.

$$
\mathbf{a}_{C o M}=\frac{\mathbf{F}_{t}}{m_{\text {body }}}+\mathbf{g}
$$

Here, $\mathbf{F}_{t}$ is the net force acting on the body, and $\mathbf{g}$ is the gravitational acceleration. The CoM position was derived from integrating the $\mathbf{a}_{C o M}$ twice. This results in $\mathbf{x}_{C o M \text {,int }}$ which was high pass filtered with a cut off at $0.4 \mathrm{~Hz}$ to obtain $\mathbf{x}_{C o M, H F}$. This is the same cut off as that of Stage LF low pass filter. The $\mathbf{x}_{C o M, L F}$ and $\mathbf{x}_{C o M, H F}$ were fused using a complementary filter to obtain the trajectory of CoM. Fig. 2 shows the extrapolated center of mass (XCoM) that can be obtained by the following equation.

$$
X C o M=C o M+\frac{\mathbf{v}_{C o M}}{\omega_{0}}
$$

Here $\mathbf{v}_{C o M}$ is the velocity of CoM and an indicator of the direction of movement, $\omega_{0}$ is given as $\sqrt{g / l_{0}}$, where $g=9.8$ $\mathrm{m} / \mathrm{s}^{2}$ (gravity), and $l_{0}$ is the vertical CoM position [10], [11]. Fig. 2 shows the dynamic Margin of Stability (MoS) as the shortest distance between the vertical projection of XCoM on the ground (XCoM') from the frontline of BoS [9].

\section{E. Estimation of $3 D F \& M$ from Pressure Insoles}

The AGBS provides positions, and 3D F\&M of the feet. This is used to estimate CoM, XCoM, and MoS as shown earlier. However, pressure insoles are only able to provide the 1D plantar pressures. Therefore, subject specific models (SSRM) were created to estimate 3D F\&M from 1D plantar pressure data.

First, the data from IMUs, 3D F\&M, and pressure insoles were synchronized. Trials that had sensor issues or measurement errors were removed. It was made sure that each subject had at least three trials for each walking task. Fig. 3(a) shows the workflow of building an SSRM. First, all 


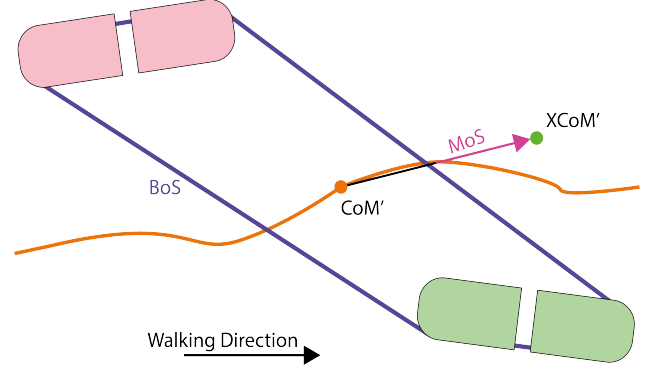

Fig. 2. Visual representation of the projected Centre of Mass (CoM'), projected Extrapolated Centre of Mass (XCoM'), and dynamic Margin of Stability (MoS). The orange line is the trajectory of CoM. The blue lines denote the borders of the Base of Support $(\mathrm{BoS})$. The pink arrow denotes the length of the MoS, measured from the frontline of the BoS to the XCoM'.

walking trials from the Normal, Slow, and Very Slow tasks are appended. This forms one extended dataset containing 1D pressures for all walking trials with all speeds as inputs for the SSRM. Then, the SSRM is built as a linear regression model fitted between the inputs and targets (3D F\&M) using least squares method. This results in six different models, one for each dimension of the F\&M. The SSRM built using the walking profiles for three different speeds is then used to estimate the 3D F\&M for each walking speed separately, and also for the Bag task which was not included in building the SSRM. Walking velocity was not used as an input to the SSRM. The same model can predict forces and moments during quiet standing, initiation of walking, cyclical walking and deceleration of walking and stopping for different walking speeds. The modelling process was repeated to create SSRMs for each subject. Fig. 3(b) shows the sensors used for the two stages, Stage LF and HF, and how the 3D F\&M estimated using the SSRMs was used to calculate the CoM (along with foot positions), XCoM, and MoS. These measures were then compared with the measurements from the AGBS.

\section{F. Influence of Sensor Choice}

The influence of the sensor configuration on the estimation of CoM, XCoM, and MoS was studied next. By reducing the number of pressure sensors used, the contribution of foot kinematics and kinetics can be understood better. Additionally, this could serve as a reference to design sensor setups with required error margins of stability parameters. Therefore, the number of pressure inputs used in building the SSRMs is varied. Fig. 4 shows the placement of the 151 individual pressure sensors on the insole as 'pluses'. Initially, an SSRM was built using all the sensors. This was followed by building SSRMs with different sensor configurations. To do so, specific foot regions were selected using an IEE (IEE S.A, Luxembourg) pressure insole as reference. The IEE insole has 8 sensors placed strategically under four foot regions; two under the toe, three under the metatarsal, one covering the arch, and two under the heel. They can be seen in Fig. 4 as green regions. The different configurations used are described as follows.

1) All: All 151 sensors are used to build the SSRM.
2) $F F$ : The pressure sensors in the medilogic ${ }^{\circledR}$ insole that corresponded to the 8 sensor locations were used for building the SSRMs. This covered the four foot regions.

3) $T+H$ : This configuration covered only the toe and heel regions.

4) T: This configuration covered the toe region alone.

5) $H$ : This configuration covered the heel region alone.

6) None: In this the accuracy of estimating CoM, XCoM, and MoS is studied under the absence of any force or moment information. Therefore, only foot positions are used in this configuration. In order to proceed, a few assumptions are made. First, the position of the CoP during standing is assumed to lie equidistant from the center of two feet. Secondly, during single stance, the CoP lies entirely at the center of the foot in contact with the ground. Thirdly, during double stance phase, a smooth transition assumption (using spline interpolation) is used to smoothly shift weight from the lagging to the leading foot [25]. These assumptions were used in Stage LF to obtain CoM. The Stage HF estimation of the CoM is not performed as there is no data about the force acting on the body in this configuration.

\section{G. Classification of Stability}

The MoS, an indication of dynamic balance, both in anterior-posterior as well as medio-lateral directions, gives information about walking stability [10], [11]. However, here only the gross placement of the projection of $\mathrm{XCoM}$ (XCoM') in $2 \mathrm{D}$ w.r.t the frontline of the $\mathrm{BoS}$ is studied. The $\mathrm{BoS}$ is estimated using the AGBS setup. The XCoM' could then be classified as either ahead or behind the BoS. Instances when the $\mathrm{XCoM}^{\prime}$ is ahead of the BoS can be termed as unstable. This is compared between the SSRM and AGBS estimated XCoM's. This exercise is done to study how closely the SSRM based system can identify instances of instability as compared to the AGBS.

\section{H. Analysis of Results}

The root mean square (RMS) of the differences normalised to the range of measured values was calculated between estimations by SSRMs and results obtained from the AGBS. This is done for the 3D forces (rrmsdF) and moments (rrmsdM), and compared with results seen in literature. This is an estimation of the error margin present when the 3D F\&M are predicted by the SSRMs. Following this, the absolute RMS of the differences $(r m s d X)$ for the XCoM was found, which quantifies the uncertainty in measurement. Then, a study of the contribution by the two stages, Stage LF and HF, to the accuracy of the XCoM was done. In this case, the Mean Absolute Distance (madt), the 2D distance in the XY plane, is calculated. Finally, the percentage of correctly classified (PCC) samples of stability by the SSRMs was studied.

\section{RESULTS}

\section{A. Forces and Moments}

The mean (standard deviations) walking speeds of the subjects for the Normal, Slow, V Slow, and Bag walking tasks 


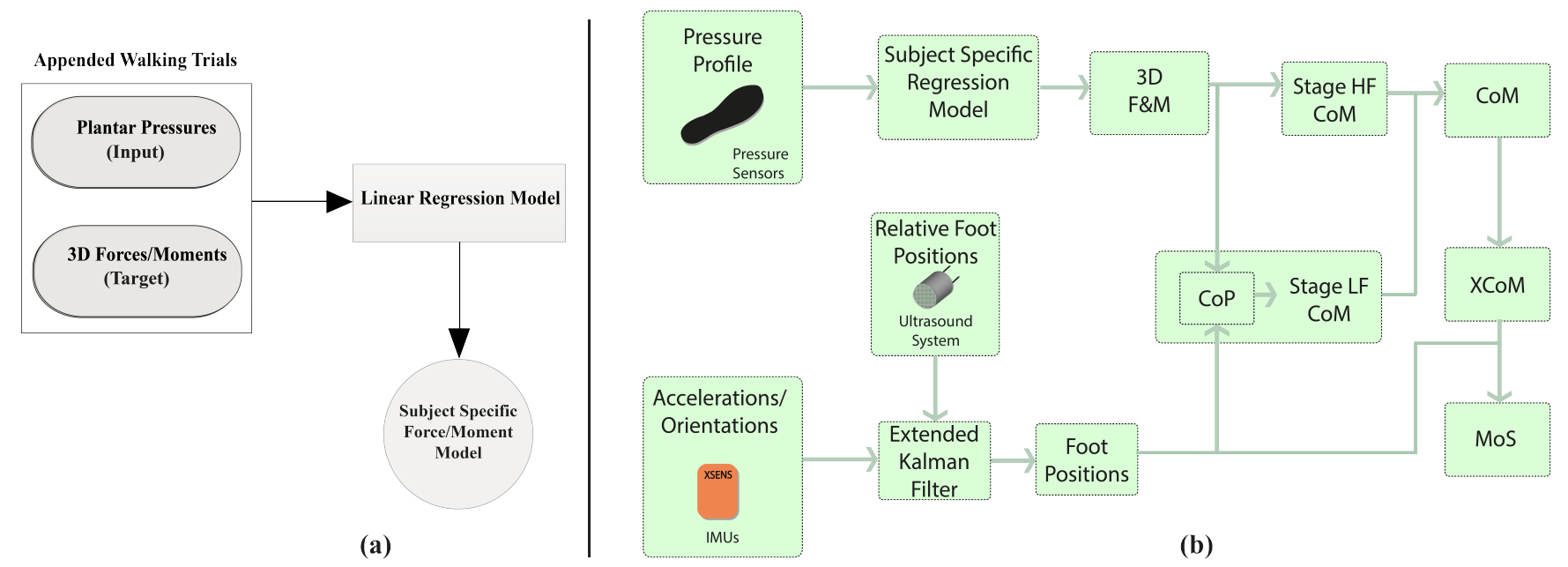

Fig. 3. (a) All Normal, Slow, and Very Slow walking trials per subject are appended before training a subject specific regression model. (b) Workflow capturing the sensors and algorithms used to estimate the Centre of Mass (CoM), Extrapolated Centre of Mass (XCoM), and dynamic Margin of Stability (MoS) when using pressure sensors.

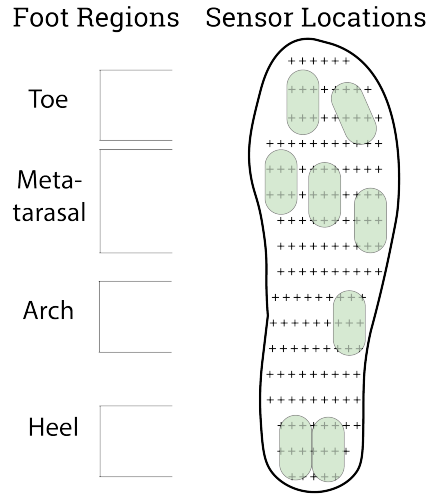

Fig. 4. The four foot regions are depicted here. The pluses denote the medilogic ${ }^{\circledR}$ pressure sensors. The shaded regions denote the sensors of IEE. The sensors in the medilogic ${ }^{\circledR}$ insole that correspond to the shaded regions are used for analysis.

are $1.03(0.15), 0.6(0.1), 0.42(0.05)$, and $0.98(0.2) \mathrm{m} / \mathrm{s}$ respectively. The regression models were built using walking trials in the Normal, Slow, and Very Slow tasks. The total number of steps (averaged between left and right) for each of the six subjects used in building the SSRMs were 97, $100,116,133,162$, and 200 . The number of steps vary as each subject differed in number of valid trials used, and also step sizes. Fig. 5(a) shows the comparison between the 3D forces estimated by the SSRM and measured by the 3D F\&M sensors (of the AGBS). The mean values of each subject is displayed as a boxplot. The distributions are shown for the different walking tasks for each sensor configuration ('All', ' $\mathrm{T}+\mathrm{H}$ ', 'H', 'T') used in the SSRM. The values are averaged over the left and right leg. In the figures, the white filled circle denotes the inter-subject mean of the distribution. The filled box contains the distribution that lies between $25 \%$ to $75 \%$ of the data. The outliers of the distribution beyond 1.5 times the interquartile range is denoted by stars.

In Fig. 5(a), the forces estimated by the SSRM built with 'All' sensors shows relatively small error margins with the
AGBS measured forces. This is seen to be the case for Normal, Slow, and Very Slow tasks. This shows that the model can be applied to different walking speeds, making it robust to changes in walking speeds. The SSRMs were used to estimate the forces in the Bag walking task to test its validity in an untrained scenario. The Bag task involves walking at the preferred walking speed while carrying a bag weighing $5 \mathrm{~kg}$. Fig. 5(a) shows that when using 'All' sensors, the forces can be estimated accurately for the untrained Bag task. The rrmsdF in all three axes is $6 \%$ or less. This shows that the model is not only capable of reproducing the forces during an untrained walking profile, but can also be used when the subject is carrying a small additional load. This could be due to the subject specificity of the SSRMs, enabling it to map the walking profile of each subject under different conditions.

The estimations remain relatively accurate when we consider the 'FF' sensor configuration. The rrmsdF is below $12 \%$ in all the three axes, for all walking tasks. Other sensor configurations show decreasing correlation coefficients and increasing RMS errors when reducing number of sensors for all walking tasks. When considering the ' $\mathrm{T}$ ' or ' $\mathrm{H}$ ' sensor configuration, we see that the error margin in rrms $d F$ increases to $20 \%$. This is expected as these configurations do not contain pressure profile during either the toe off or heel strike. Additionally, as the number of sensors are reduced, the regression model uses lesser sensors to estimate the forces. Fig. 5(b) shows the comparison between the 3D moments estimated by the SSRM and measured by the AGBS. Similar results as seen in forces can be observed in the estimation of moments. Good accuracy in estimating the moments is seen while using the 'All' sensor configuration for Normal, Slow, and Very Slow tasks, and also for the untrained Bag task. Moments, however, show larger errors than forces as they are a cross product of distance and forces in the two orthogonal axes. Subsequently, the estimation worsens as the number of sensors are reduced. In both Fig. 5(a) and (b), the results for 'None' sensor configuration is not shown as there is no force 


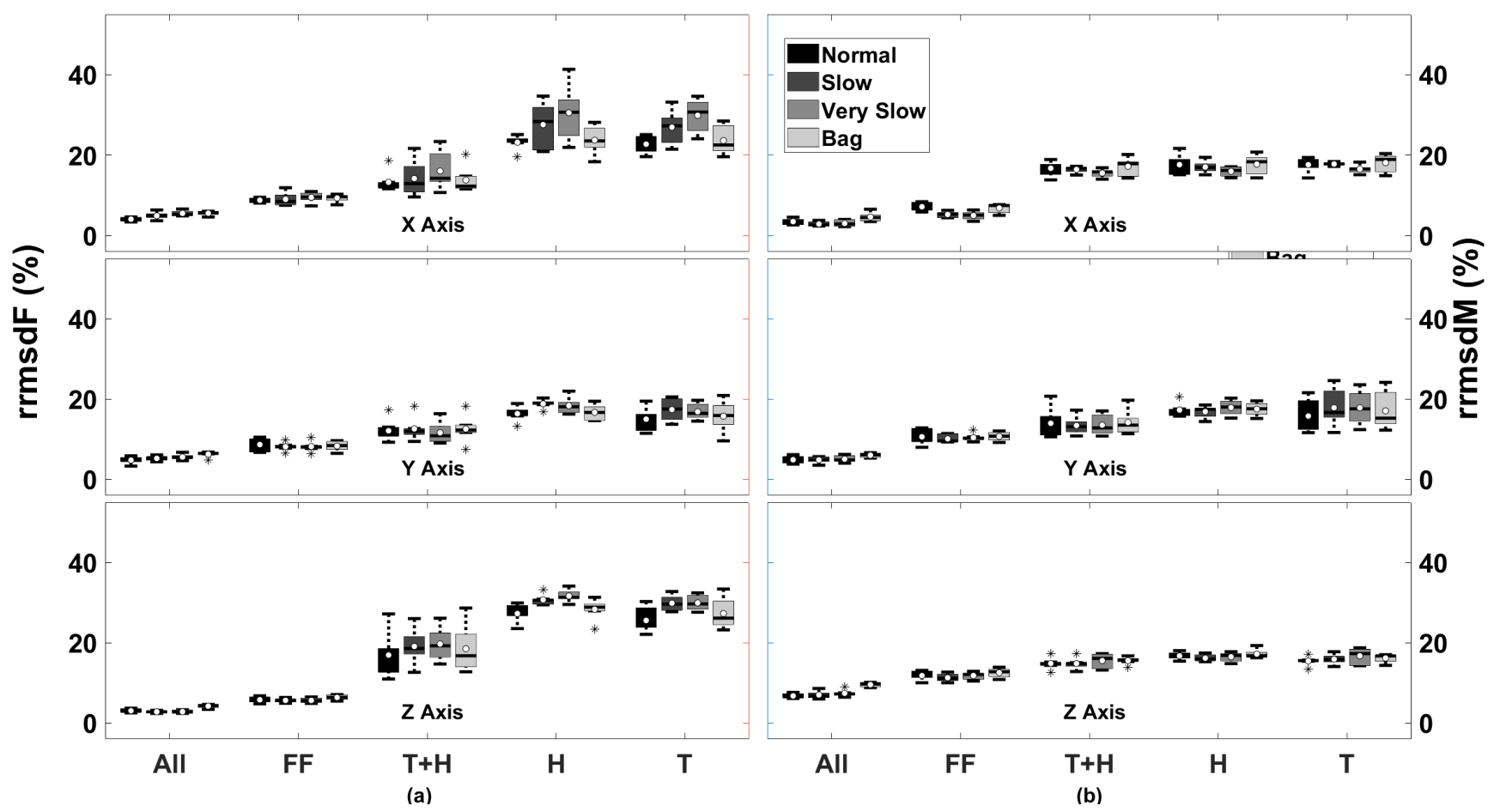

Fig. 5. (a) shows the range normalised RMS ( $r r m s d F$ ) between the forces, and (b) the range normalised RMS (rrmsdM) between the moments, estimated using SSRMs and measured from AGBS. Box plots of the mean values are shown for the different walking tasks for each sensor configuration used: All (All), Four foot regions (FF), Toe+Heel $(\mathrm{T}+\mathrm{H})$, Toe(T), and Heel(H).

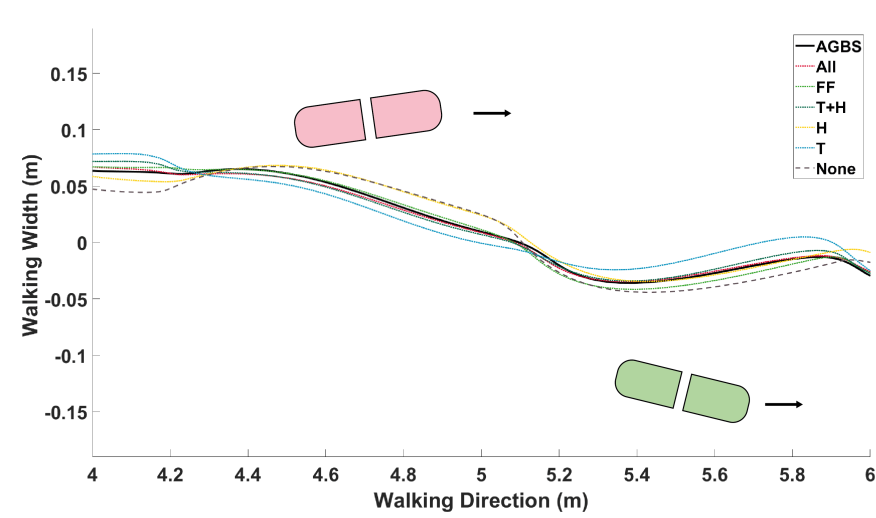

(a)

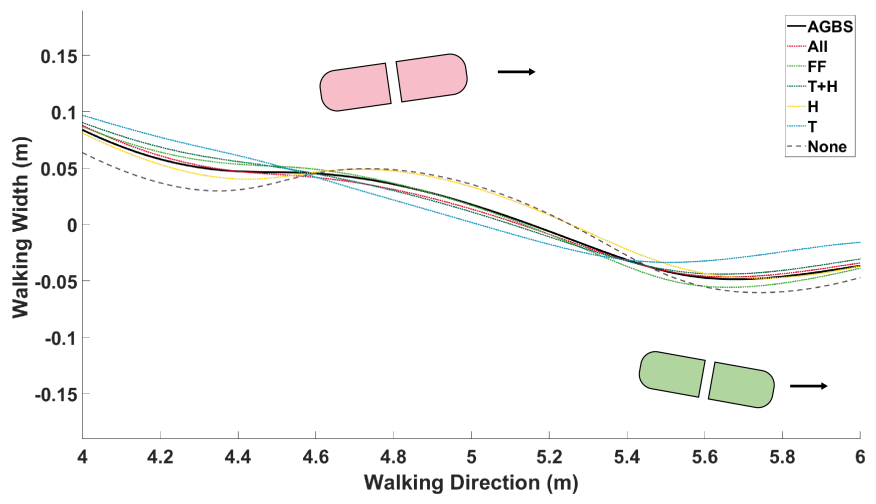

(b)

Fig. 6. Representative trajectory of (a) CoM and (b) XCoM from a Normal walking task. The black line shows the reference trajectory estimated using the AGBS. The other dotted lines show the trajectory with respect to different sensor configurations. The feet of the subject is shown in pink and green.

or moment information in these cases.

\section{B. Dynamic Balance}

The 3D F\&M from AGBS and SSRM are then used to estimate the CoM using Stage LF and Stage HF. Fig. 6 shows representative trajectories of the CoM and XCoM for a Normal task. The CoM fluctuates between the two feet as the stance changes. Simultaneously, in 6(b) the XCoM can be seen to oscillate with changes in the instantaneous velocity of the CoM. The SSRM trajectories deviate from the reference trajectory estimated by the AGBS, as they have different accuracies in estimating the forces and moments. Fig. 7 shows the comparison of $\mathrm{XCOM}$ in only the $\mathrm{X}$ and $\mathrm{Y}$ axes as we are interested only in the projection of XCoM, i.e., XCoM' on the ground as rmsdX. When using 'All' sensors, the accuracy of estimating XCoM' for all walking tasks is high. The rmsdX is less than $2 \mathrm{~cm}$ for the $\mathrm{X}$ and $\mathrm{Y}$ axes respectively. This is the case for all walking tasks. For other sensor configurations (' $\mathrm{T}+\mathrm{H}$ ', ' $\mathrm{T}$ ', and ' $\mathrm{H}$ '), the $r m s d X$ does not change drastically as seen with $3 \mathrm{D}$ F\&M in fig. 5(a) or (b). Although there is no force or moment information of the feet in the 'None' configuration, the XCoM' has low errors in both $\mathrm{X}$ and $\mathrm{Y}$ axes. The $r m s d X$ for the $\mathrm{X}$ and $\mathrm{Y}$ axis is less than 12 and $8 \mathrm{~cm}$ respectively. Assuming step width during quiet stance as $15 \mathrm{~cm}$, maximum step width and length as $40 \mathrm{~cm}$ and $70 \mathrm{~cm}$ respectively, the BoS can range between $700 \mathrm{~cm}^{2}$ to a maximum of 


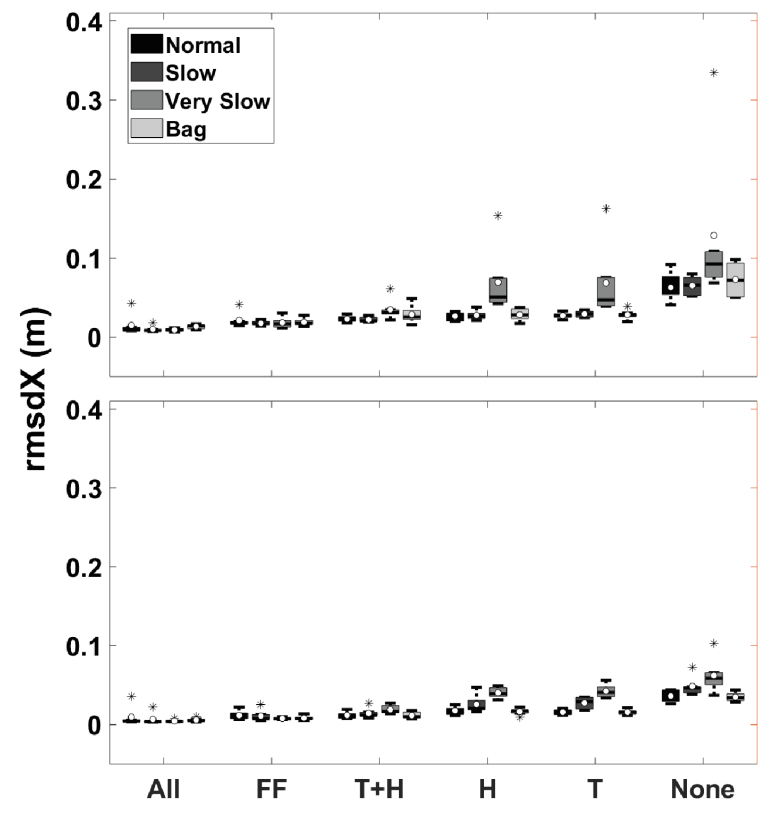

Fig. 7. RMS of the differences between the XCoM estimated using regression model and measured from AGBS (rmsdX). Box plots of the mean values are shown for the different walking tasks for each sensor configuration used.

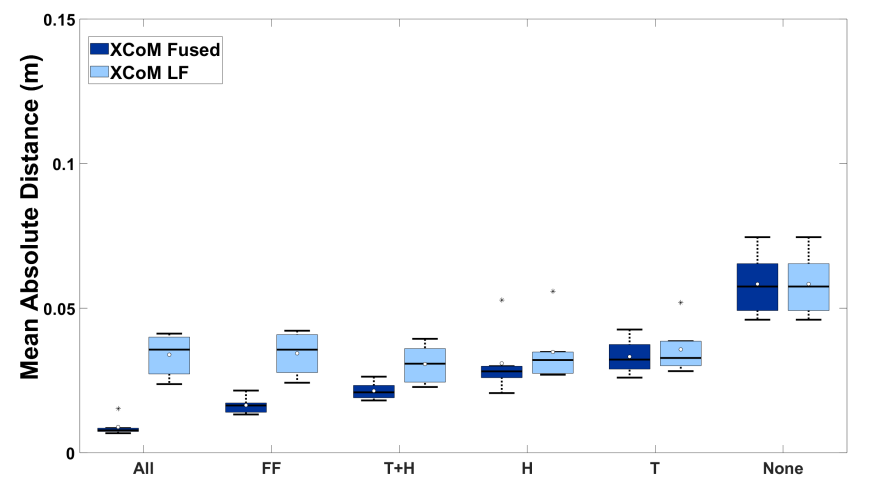

Fig. 8. Error between XCoM with both low and high frequency (XCoM fused) and XCoM with only low frequency content (XCoM LF) for the Slow task.

$2400 \mathrm{~cm}^{2}$. Comparing error margins of XCoM with respect to the BoS gives an idea about the relative magnitude of the error.

The previous paragraph threw light on the influence of $3 \mathrm{D}$ F\&M estimation accuracy on the estimation of XCoM. Next, the contribution of Stage $\mathrm{HF}$ on the estimation of XCoM is studied. Here, we hypothesize that for lower walking speeds, there is lesser useful information in the higher frequencies. This is acceptable as our primary applications are towards people with gait impairment. Also, the Stage HF relies on the need for good kinetic information. Lesser reliance on this translates to the possibility of eliminating kinetic sensors. Therefore, the XCoM' with and without the Stage HF is considered. Then, the madt is calculated between the estimations by the SSRMs and AGBS. Fig. 8 shows the distribution of madt in two cases during the Slow task. One case (XCoM Fused) contains the madt between the fused XCoM' from the SSRM from the AGBS. The second case (XCoM LF) contains the madt between the XCoM' with only low frequency information and the fused XCoM' of the AGBS. In Fig. 8, the XCoM Fused shows lower madt than XCoM LF when compared with the estimations from the AGBS. The average madt for XCoM LF in all sensor configurations (except 'None') is about $3 \mathrm{~cm}$. The distribution of the madt for XCoM LF shows larger variance, but remains robust with reduction in sensor configuration. This suggests that for walking at low speeds, the low frequency information of CoM is sufficient to estimate XCoM given an error margin of $3 \mathrm{~cm}$. This could translate to advantages in terms of lower sampling rates of sensors, which may influence power consumption, or the possibility to eliminate kinetic sensors. Note that in case of 'None', the XCoM LF and XCoM Fused distributions are similar as this sensor configuration does not have any Stage HF information.

\section{Classification of Stability}

Once the XCoM' estimated by the SSRMs is classified as ahead or behind the frontline of the BoS, it is compared with the classifications done by the AGBS, which is assumed to be the ground truth. Fig. 9 shows the percentage of sample points rightly classified as ahead (PCC Ahead) or behind (PCC Behind) the frontline of the BoS.

In order to classify, the frontline of the BoS is needed, which is measured only during quiet standing or instances of double support. During quiet standing, the XCoM' lies well within the $\mathrm{BoS}$, as there is no movement. During these instances, XCoM' is most correctly classified. It can be seen from Fig. 9(a) that the percentages are very high for any sensor configuration in all walking tasks. The inset table shows the percentage of actual time behind or ahead the BoS. Simultaneously, during double support phase the XCoM' is usually 'ahead' of the BoS in healthy people. However, as double support phases are very short in a gait cycle, the number of samples where the XCoM' is ahead of the BoS is limited. Subsequently, there is a higher chance of error or mismatch in the placement of XCoM' ahead of the BoS. Fig. 9(b) shows that the distributions are wider and the mean PCC is lower as compared to Fig. 9(a). For 'All' sensors, the figure shows that the SSRMs has high PCC in estimating the dynamic stability. The mean PCC is above 95\% (for both ahead and behind) for all four walking tasks. In Fig. 9, the PCC reduces as the number of sensors are reduced.

\section{DISCUSSION}

In this study, subject specific models were built instead of a generic model for all subjects. This choice was made as preliminary studies showed poor performance of a generic regression model built using trials from different subjects. Additionally, this method could be expected to perform better for people with gait impairment. Correlations between the estimated and measured 3D F\&M as average percentages among both foot were estimated, and compared with Sim et al. [19] and tabulated in Table I. Their study used fast, normal, and slow walking speeds for training a wavelet neural network that estimated the 3D F\&M from plantar pressures. It is seen that in both, this study and Sim et al., forces in the $\mathrm{Z}$ axis show the highest correlation, followed by the $\mathrm{X}$ axis. This 


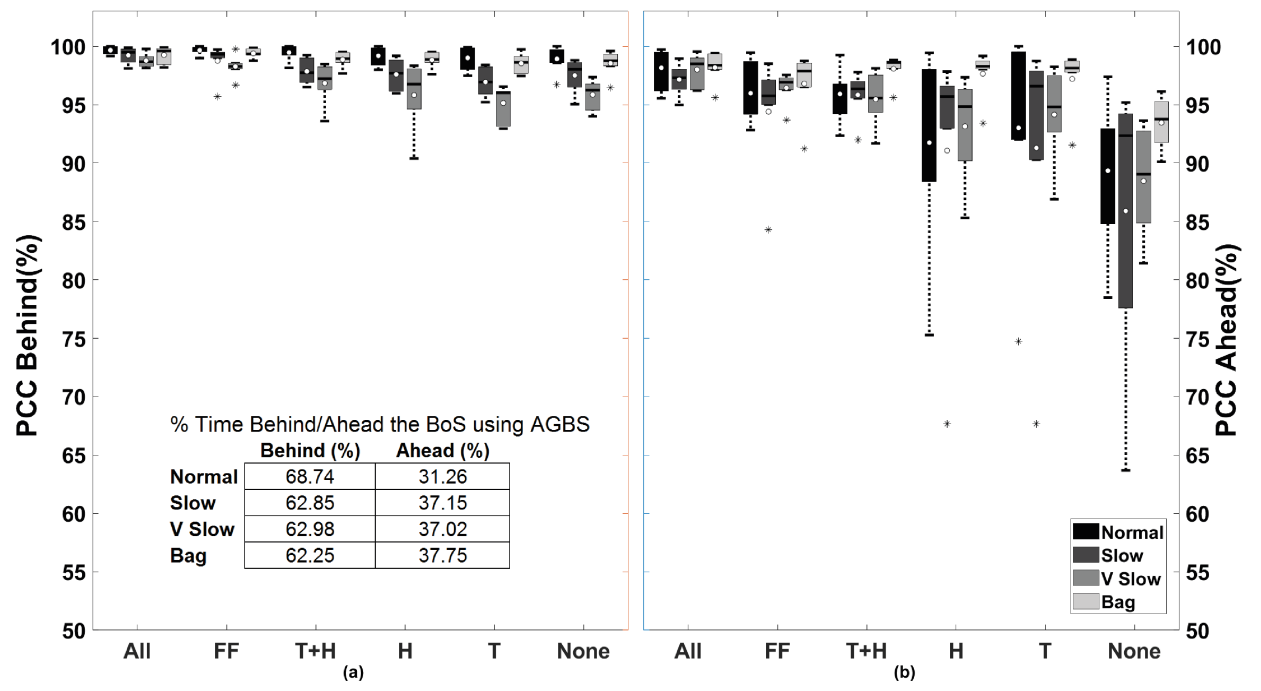

Fig. 9. Classification of XCoM' as (a) Behind or (b) Ahead of the BoS: The percentage of total time when the XCoM' is correctly classified is displayed as a box plot distribution. The classifications by the AGBS are considered to be true and used as the reference. The percentage of time ahead or behind the $\mathrm{BoS}$ as measured by AGBS is shown in the inset table.

TABLE I

COMPARISON OF THE CORRELATIONS FOUND IN THIS STUDY AND SIM $e t$ al. [19]

\begin{tabular}{|l|c|c|}
\hline & This Study (\%) & Sim et al. $\mathbf{( \% )}$ \\
\hline$F_{X}$ & 95.5 & 97.6 \\
\hline$F_{Y}$ & 95.3 & 85.3 \\
\hline$F_{Z}$ & 99.6 & 98.8 \\
\hline$M_{X}$ & 97.9 & 87 \\
\hline$M_{Y}$ & 96.3 & 88.1 \\
\hline$M_{Z}$ & 87.1 & 84.7 \\
\hline
\end{tabular}

is expected as plantar pressures are defined by the vertical ground reaction force. In case of moments, $\mathrm{Z}$ axis showed the least correlation. Comparatively, except for forces in $\mathrm{X}$ axis, this study shows slightly higher correlations with the reference, than that in Sim et al. [19]. Note that the current study doesn't use a normalised gait cycle for building the SSRMs, as Sim et al. [19] does. Therefore, the SSRMs contain information of the gait cycle during initiation and decelerations, allowing better prediction even when making short steps or shuffling at home.

The most interesting observation is found when we study the influence of $\mathrm{F} \& \mathrm{M}$ estimation on the estimation of XCoM'. We know from fig. 5, that the estimation of F\&M deteriorates as we reduce the number of sensors. However, fig. 7 shows that this has little effect on the estimation of XCoM'. It has to be noted that the foot position used is the same for all sensor configurations. This could give rise to the argument that accuracy of foot position is more important than force or moment sensing, highlighting the relative influence of foot kinetics and kinematics towards accuracy of XCoM. This argument is strengthened when considering the 'None' configuration. We see low rmsdX values in fig. 7 for this configuration. However, it must be noted that some of the assumptions, especially the smooth transition assumption, in
'None' may fail during instances of daily life such as shuffling of feet, or while turning. Nonetheless, the comparisons show that in order to identify the XCoM', we must have good accuracy in estimating the foot position. Currently, EKF and ultrasound range updates are used to improve the foot position estimation provided by the IMUs. Fig. 9 can serve as a reference for choosing the right sensor configuration based on the required task. If we are to consider an 'optimal' case that has less number of sensors while providing good accuracy, the 'FF' sensor configuration seems to be the right choice. However, the ' $\mathrm{T}+\mathrm{H}$ ' sensor configuration can be used if a more minimal setup is preferred. This configuration has only sensors under the heel and toe, but the model shows good accuracy in estimating the XCoM'. This shows that for minimal sensing of ADL, a simple sensor set with at least two pressure sensors (one under the heel and other under the toe), IMUs on the forefoot, and updates of the distance between the two feet is required for a confident estimation of the XCoM and dynamic stability.

\section{A. Limitations and Future Work}

Though the recruitment was not selective to sole size or gender, the participating group is not very diverse. There is only one shoe size tested and only one female subject in the study. However, this would not have any effect on validity of the method as the models are subject specific. Also, as the study was performed on healthy subjects, the results might vary in populations with gait impairment.

Walking trajectories were required to create the SSRMs, which in practice, would translate to a calibration phase where the subject should walk a few times using the measurement setups. As seen earlier, an average of 134 steps is needed to achieve the results shown. Though this is an extra effort, it eliminates the use of bulky 3D F\&M sensors in daily life which can modify gait pattern and cause discomfort during extended wear [7], [9]. Alternatives 
to create subject specific models could be building models generic to any subject and calibrating them before use.

In this study, the SSRMs are linear regression models. Studies have shown other possibilities for improving accuracy in predicting 3D forces/moments [20]-[22]. Further, this study finds that Stage LF is sufficient for CoM estimation. Therefore the CoP, essential for estimating CoM in Stage LF, can be found using only the pressure sensors [26]. This eliminates the conversion from 1D pressure to 3D F\&M, thereby reducing the complexity in generating SSRMs.

The subjects were asked to walk in straight lines in this study. Shuffling or turning can introduce shear forces directed towards the direction of turning. The accuracy of the SSRMs in these conditions should be studied further. Additionally, the study is missing assessments of MoS in the medio-lateral direction as it is highly relevant in understanding gait stability [12]. This study shows the feasibility of pressure sensors over 3D F\&M sensors. The IMUs can be embedded inside the insoles, as designed by Moticon ${ }^{\circledR}$ [27], allowing design for a thinner, and wearable AGBS. However, the ultrasound range estimator requires further improvement. The range estimator requires synchronized receiver and transmitter placed in a direct line of sight. Alternatives, such as using infrared, may contribute towards the design of an inconspicuous AGBS [28].

\section{COnClusions}

The AGBS in this study can thus be replaced with a setup containing pressure sensors and IMUs on each foot, and an ultrasound range estimator. During straight line walking, pressure sensors under the toe and heel can be sufficient (along with foot positions), to study the XCoM and dynamic stability of a healthy subject. Also, low frequency information of the $\mathrm{CoM}$ is sufficient for estimating the XCoM trajectory. Using a few assumptions, XCoM could also be estimated using only the estimations of foot position, ignoring any kinetic information. In addition, the current study highlights the contribution of foot kinematics and kinetics while estimating XCoM. These results could be used in the design of a lighter and wearable AGBS system. Such a system can contribute towards objective quantification of gait and balance quality in an ambulatory setup. This, unlike current clinical outcomes, can help monitor functional recovery.

\section{ACKNOWLEDGMENT}

We thank Leendert Schaake (Roessingh Research and Development), Ed Droog, and Marcel Weusthof (Universiteit Twente), for assisting with the pressure sensors and AGBS.

\section{REFERENCES}

[1] B. H. Dobkin, "Rehabilitation after stroke," New Engl. J. Medicine, vol. 352, no. 16, pp. 1677-1684, 2005.

[2] World Health Organization, Towards a Common Language for Functioning, Disability and Health. 2002.

[3] G. Kwakkel et al., "Standardized measurement of sensorimotor recovery in stroke trials: Consensus-based core recommendations from the Stroke Recovery and Rehabilitation Roundtable," Int. J. Stroke, vol. 12, no. 5, pp. 451-461, 2017.

[4] P. H. Veltink, C. Liedtke, E. Droog, and H. Van Der Kooij, "Ambulatory measurement of ground reaction forces," IEEE Trans. Neural Syst. Rehabil. Eng., vol. 13, no. 3, pp. 423-427, 2005.
[5] D. Weenk, D. Roetenberg, B.-J. F. van Beijnum, H. Hermens, and P. H. Veltink, "Ambulatory estimation of relative foot positions by fusing ultrasound and inertial sensor data," IEEE Trans. Neural Syst. Rehabil. Eng., vol. 23, no. 5, pp. 817-826, 2015.

[6] H. M. Schepers, E. van Asseldonk, J. Buurke, and P. Veltink, "Ambulatory Estimation of Center of Mass Displacement During Walking," IEEE Trans. Biomed. Eng., vol. 56, no. 4, pp. 1189-1195, 2009.

[7] C. Liedtke, S. A. W. Fokkenrood, J. T. Menger, H. van der Kooij, and P. H. Veltink, "Evaluation of instrumented shoes for ambulatory assessment of ground reaction forces," Gait Posture, vol. 26, no. 1, pp. 39-47, 2007.

[8] H. M. Schepers, H. F. J. M. Koopman, and P. H. Veltink, "Ambulatory assessment of ankle and foot dynamics," IEEE Trans. Biomed. Eng., vol. 54, no. 5, pp. 895-902, 2007.

[9] F. B. van Meulen, D. Weenk, J. H. Buurke, B. F. van Beijnum, and P. H. Veltink, "Ambulatory assessment of walking balance after stroke using instrumented shoes," J. Neuroeng. Rehabil., vol. 13, no. 1, p. 10, 2016.

[10] F. B. van Meulen, D. Weenk, E. H. F. van Asseldonk, H. M. Schepers, P. H. Veltink, and J. H. Buurke, "Analysis of balance during functional walking in stroke survivors," Plos One, vol. 11, no. 11, p. 20, 2016.

[11] A. L. Hof, M. G. J. Gazendam, and W. E. Sinke, "The condition for dynamic stability," J. Biomech., vol. 38, no. 1, pp. 1-8, 2005.

[12] S. M. Bruijn, O. G. Meijer, P. J. Beek, and J. H. van Dieën, "Assessing the stability of human locomotion: a review of current measures.," $J$. Royal Soc. Interface / Royal Soc., vol. 10, no. 83, p. $20120999,2013$.

[13] D. Roetenberg, H. Luinge, and P. Slycke, "Xsens MVN : Full 6DOF Human Motion Tracking Xsens MVN : Full 6DOF Human Motion Tracking Using Miniature Inertial Sensors," 2009.

[14] A. Karatsidis et al., "Estimation of Ground Reaction Forces and Moments During Gait Using Only Inertial Motion Capture," Sensors, vol. 17 , no. 1 , p. $75,2016$.

[15] N. Hegde, M. Bries, and E. Sazonov, "A Comparative Review of Footwear-Based Wearable Systems," Electron., vol. 5, p. 28, aug 2016.

[16] A. H. Abdul Razak, A. Zayegh, R. K. Begg, and Y. Wahab, "Foot plantar pressure measurement system: A Review," Sensors, vol. 12, no. 12, pp. 9884-9912, 2012.

[17] M. Koch, L. K. Lunde, M. Ernst, S. Knardahl, and K. B. Veiersted, "Validity and reliability of pressure-measurement insoles for vertical ground reaction force assessment in field situations," Appl. Ergonomics, vol. 53, pp. 44-51, 2016.

[18] A. Forner Cordero, H. J. F. M. Koopman, and F. C. T. Van Der Helm, "Use of pressure insoles to calculate the complete ground reaction forces," J. Biomech., vol. 37, no. 9, pp. 1427-1432, 2004.

[19] T. Sim et al., "Predicting complete ground reaction forces and moments during gait with insole plantar pressure information using a wavelet neural network," J. Biomech. Eng., vol. 137, pp. 1-9, 2015.

[20] H. Savelberg and A. de Lange, "Assessment of the horizontal , fore-aft component of the ground reaction force from insole pressure patterns by using artificial neural," Clin. Biomech., vol. 14, pp. 585-592, 1999.

[21] D. T. P. Fong, Y. Y. Chan, Y. Hong, P. S. H. Yung, K. Y. Fung, and K. M. Chan, "Estimating the complete ground reaction forces with pressure insoles in walking," J. Biomech., vol. 41, no. 11, pp. 2597-2601, 2008.

[22] H. Rouhani, J. Favre, X. Crevoisier, and K. Aminian, "Ambulatory assessment of 3D ground reaction force using plantar pressure distribution," Gait Posture, vol. 32, no. 3, pp. 311-316, 2010.

[23] M. I. Mohamed Refai, B.-J. F. van Beijnum, J. H. Buurke, H. Koopman, and P. H. Veltink, "Pressure Insoles for Gait and Balance Estimation," Master's thesis, Universiteit Twente, The Netherlands, 2017.

[24] A. L. Hof, "Scaling gait data to body size," Gait Posture, vol. 4, no. 3, pp. 222-223, 1996.

[25] L. Ren, R. K. Jones, and D. Howard, "Whole body inverse dynamics over a complete gait cycle based only on measured kinematics," $J$. Biomech., vol. 41, no. 12, pp. 2750-2759, 2008.

[26] M. I. Mohamed Refai, B.-J. F. van Beijnum, J. Buurke, and P. Veltink, "Ambulatory estimation of xcom using pressure insoles and imus," in Proceedings of 3D Analysis of Human Movement Conference, pp. 109$110,2018$.

[27] "Moticon insoles.". Available: https://www.moticon.de/ (Date last accessed 29-June-2018).

[28] S. Bertuletti, A. Cereatti, M. Caldara, and U. D. Croce, "A proximity sensor for the measurement of the inter-foot distance in static and dynamic tasks," Gait Posture, vol. 49, no. 2016, p. S15, 2016. 


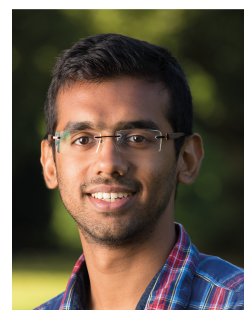

Mohamed Irfan Mohamed Refai received the M.Sc. degree in Electrical Engineering with Research Honors in 2017 from the Universiteit Twente, The Netherlands. $\mathrm{He}$ is currently pursuing a $\mathrm{PhD}$ degree at the Biomedical Signals and Systems group in the same university since 2017 . His research, as part of the NeuroCIMT project, aims at developing an on-body sensing and feedback system for patienttailored motor rehabilitation.

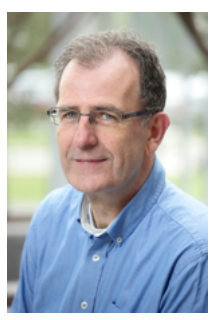

Bert-Jan F. van Beijnum (M'14) received the M.Sc. and Ph.D. degrees in Electrical Engineering from the Universiteit Twente. He is an Associate Professor in the Biomedical Signals and Systems group at the Universiteit Twente. His research is embedded in projects of the Digital Society Institute and TechMed. His interests include telemedicine, mobile virtual communities for health, smart health systems, smart technologies for decision support, feedback, and coaching.

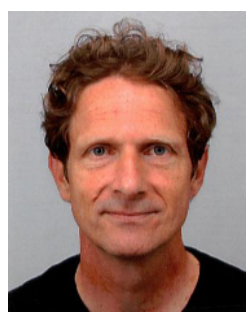

Jaap H. Buurke received his $\mathrm{PhD}$ from the Universiteit Twente. $\mathrm{He}$ is coordinator of the $\mathrm{Re}$ search Track Rehabilitation Technology at Roessingh Research and Development, adjunct professor at Northwestern University Chicago (USA), senior researcher at Roessingh, Centre for Rehabilitation and full Professor at Biomedical Signals and Systems group of the Universiteit Twente. Since 2011, he is the president of the Dutch society for Neurorehabilitation. He is specialised in human movement analysis with specific expertise in neuromuscular control and biomechanics after stroke. He is actively involved in several projects focusing on motor control, movement analysis, rehabilitation robotics and active assistive devices.

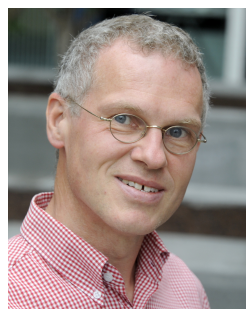

Peter H. Veltink (SM'06) is Professor of Technology for the restoration of human function at the Biomedical signals and Systems group, Universiteit Twente. His research interests are in biomechatronics and neural engineering. His research includes ambulatory sensing of human movements, artificial human motor control, and neurostimulation. $\mathrm{He}$ is the (co)author of over 125 peer reviewed journal papers. He has been an Associate Editor for the IEEE Transactions on Neural Systems and Rehabilitation Engineering until 2014. 